\title{
Cerebral Venous Sinus Thrombosis in Patients With SARS-CoV-2
}

\author{
Matthew Fitzsimons ${ }^{\mathrm{a}}$, Michael C. Li ${ }^{\mathrm{a}}$, Abinayaa Ravichandran ${ }^{\mathrm{b}}$, Ramiro G. Castro Apolo ${ }^{\mathrm{b}}$, \\ Erafat Rehim ${ }^{b}$, Hussam A. Yacoub ${ }^{a, b, c}$
}

\begin{abstract}
Several neurological manifestations of the severe acute respiratory syndrome coronavirus 2 (SARS-CoV-2) have been reported, including cerebral venous sinus thrombosis (CVST). In this report, we discuss two cases of cerebral venous thrombosis in young patients with a recent SARS-CoV-2 infection and otherwise unrevealing workup. Case 1 was a 35-year-old man who was found unresponsive with urinary incontinence and vomitus on the floor. Computed tomography (CT) angiogram of the head revealed evidence of extensive venous thrombosis. Extensive workup for thrombotic disease was unrevealing and SARS-CoV-2 was detected via polymerase chain reaction. Anticoagulation was immediately initiated, and the patient made a good clinical recovery. Case 2 was a 31-year-old man who presented with severe decreased level of consciousness and was found to have extensive cerebral sinus thrombosis. Past medical history was significant for a recent SARS-CoV-2 infection diagnosed 12 days prior. The patient was treated with intravenous heparin and eventually oral anticoagulation with good clinical outcome. CVST is a potential cerebrovascular complication of SARS-CoV-2. We advise clinicians to consider this diagnosis in patients with a recent SARS-CoV-2 infection in the appropriate clinical setting.
\end{abstract}

Keywords: Cerebral venous sinus thrombosis; SARS-CoV-2; COVID-19; Stroke

\section{Introduction}

Several typical and atypical neurological manifestations of viral pandemics have been reported. Neurological manifestations of the severe acute respiratory syndrome coronavirus 2

Manuscript submitted July 20, 2021, accepted September 2, 2021

Published online October 5, 2021

${ }^{a}$ Morsani College of Medicine, University of South Florida, Tampa, FL, USA bDepartment of Neurology, Lehigh Valley Hospital and Health Network, Allentown, PA, USA

${ }^{\mathrm{c} C o r r e s p o n d i n g ~ A u t h o r: ~ H u s s a m ~ A . ~ Y a c o u b, ~ D e p a r t m e n t ~ o f ~ N e u r o l o g y, ~ L e h i g h ~}$ Valley Hospital and Health Network, 1250 South Cedar Crest Blvd, Suite 405, Allentown, PA 18103, USA. Email: hyacoub123@yahoo.com

doi: https://doi.org/10.14740/jnr697
(SARS-CoV-2) are rare. Among these are acute cerebrovascular events including ischemic stroke and cerebral venous sinus thrombosis (CVST). CVST is a rare emergency with an estimated incidence of 1.6 per 100,000 per year in one populationbased study [1]. The underlying pathophysiology of CVST in patients with SARS-CoV-2 remains to be elucidated.

In this report, we present two cases of young men who presented with decreased level of consciousness. Magnetic resonance imaging (MRI) and venography revealed extensive CVST. Both patients were diagnosed with a recent SARS$\mathrm{CoV}-2$ infection, and neither had any risk factors for venous thrombosis with both acquired and inherited thrombotic risk panels unrevealing. Both patients were treated with oral anticoagulation with good clinical outcome.

\section{Case Reports}

\section{Case 1}

A right-handed 35-year-old man presented to the emergency department (ED) after being found unresponsive with urinary incontinence and vomitus on the floor. An overdose with prescribed antidepressants was suspected and naloxone was immediately administered with no clinical improvement. Past medical history was significant for major depressive and borderline personality disorders. Social history was significant for prior use of cannabis, a 15 pack-year history of tobacco use, and current use of e-cigarettes. There was no known history of illicit drug use. The patient was an inmate in prison prior to this presentation. Review of systems could not be obtained due to deceased level of consciousness. On initial evaluation, the patient was found to be severely somnolent with a Glasgow Coma Score (GCS) of 11 and no evidence of head trauma. The patient was afebrile with oxygen saturation of $89 \%$ which improved to $95 \%$ after administration of oxygen via nasal cannula. General examination in the ED was unrevealing except for a coarse-sounding cough with rhonchi noted on pulmonary auscultation. Initial neurological evaluation revealed that patient was mute with no attempt to communicate with the examiner. Evaluation of fund of knowledge, memory, and judgement was limited. Cranial nerve examination revealed equal pupils with brisk reaction to light. Brain stem reflexes including the corneal and oculocephalic were intact. Patient was responsive only to painful stimuli and was able to move all 
extremities with spastic motor paresis but no clonus. Sensory, coordination, and gait examination could not be assessed.

Laboratory workup included a complete blood count $(\mathrm{CBC})$ revealing a white blood cell count $(\mathrm{WBC})$ of $11.9 \times$ $10^{3} / \mathrm{mm}^{3}$ (normal range: $4.5-11 \times 10^{3} / \mathrm{mm}^{3}$ ), platelet count of $403 \times 10^{3} / \mathrm{mm}^{3}$ (normal range: $140-350 \times 10^{3} / \mathrm{mm}^{3}$ ), and mean platelet volume of $7.1 \mathrm{fL}$ (normal range: 7.5 - $11.3 \mathrm{fL}$ ). Comprehensive metabolic panel (CMP) revealed a serum glucose level of $176 \mathrm{mg} / \mathrm{dL}$ (normal range: 70 - $110 \mathrm{mg} / \mathrm{dL}$ ), alkaline phosphatase of $111 \mathrm{U} / \mathrm{L}$ (normal range: 32 - $91 \mathrm{U} / \mathrm{L}$ ), anion gap 14 (normal range: 3 - 11), and total bilirubin $1.3 \mathrm{mg} / \mathrm{dL}$ (normal range: 0.2 - $1.0 \mathrm{mg} / \mathrm{dL}$ ). Additional laboratory workup revealed elevated fibrinogen of $607 \mathrm{mg} / \mathrm{dL}$ (normal range: 180 - 500), and D-dimer of $2.37 \mu \mathrm{g} / \mathrm{mL}$ FEU (normal $<0.5 \mu \mathrm{g} /$ $\mathrm{mL}$ FEU). Initial rapid coronavirus disease 2019 (COVID-19) test was negative. Other investigations which were normal included hemoglobin, hematocrit, blood urea nitrogen (BUN), serum creatinine, sodium, potassium, partial thromboplastin time, and prothrombin time.

Chest X-ray demonstrated no acute cardiopulmonary disease. Computed tomography (CT) scan of the head revealed an area of hypoattenuation in the left temporo-occipital region, within the vascular territory of the posterior cerebral artery, suggesting an acute ischemic stroke (Fig. 1). CT of the head also showed a hyperdense straight sinus consistent with cerebral venous thrombosis (Fig. 2a, b). The patient was transferred to the neurocritical care unit for further management. Magnetic resonance venography (MRV) of the head revealed extensive dural venous sinus thrombosis involving the transverse, sigmoid, and superior aspect of the internal jugular vein on the left (Fig. 3a) in addition to thrombosis of the vein of Galen and straight sinus (Fig. 3b). MRI of the brain revealed several areas of ischemia involving the thalamus bilaterally and left hippocampus/parahippocampal gyrus and occipital lobe (Fig. $4 \mathrm{a}, \mathrm{b}$ ). The patient was started on continuous intravenous (IV) heparin $(5-30 \mathrm{U} / \mathrm{kg} / \mathrm{h})$.

Continuous electroencephalogram did not show any elec-

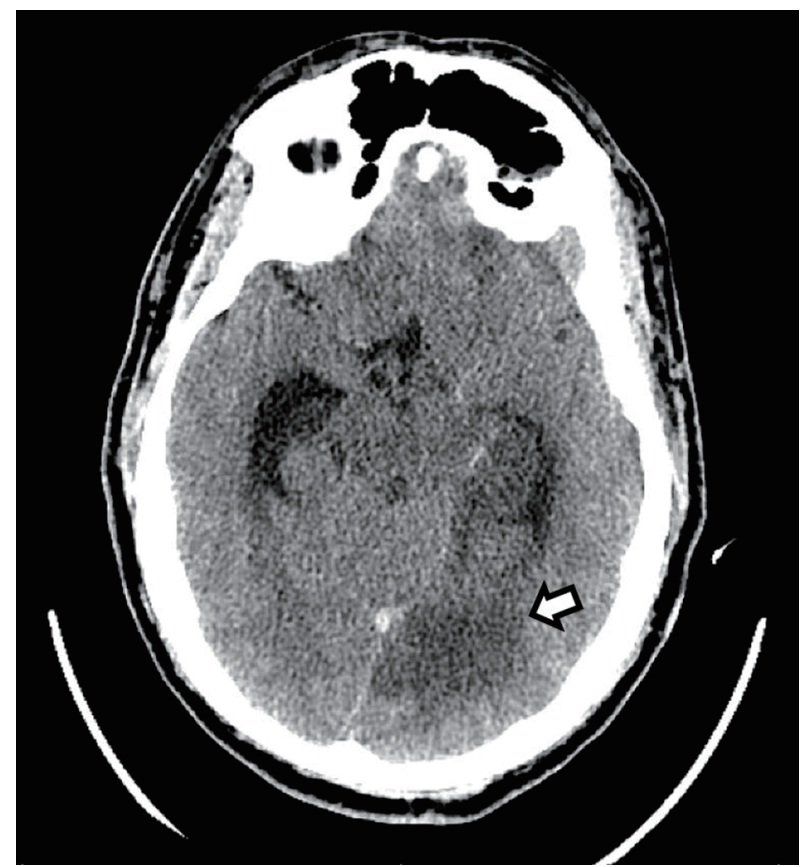

Figure 1. CT of the head without contrast shows an area of hypodensity involving the left medial temporal and occipital lobes, consistent with a subacute ischemic stroke (arrow). CT: computed tomography.

trographic evidence of seizures but revealed potential seizure foci in the left cerebral hemisphere. The patient was started on IV levetiracetam, $750 \mathrm{mg}$ every $12 \mathrm{~h}$. Given the finding of extensive cerebral venous thrombosis in the young, further workup included complete inherited and acquired thrombotic risk panel including factor $\mathrm{V}$ Leiden and prothrombin gene mutations which was unrevealing. SARS-CoV-2 was detected via polymerase chain reaction. The patient was clinically stable and eventually transferred out of the neurocritical care unit. He was transitioned successfully to oral feeds and eventually
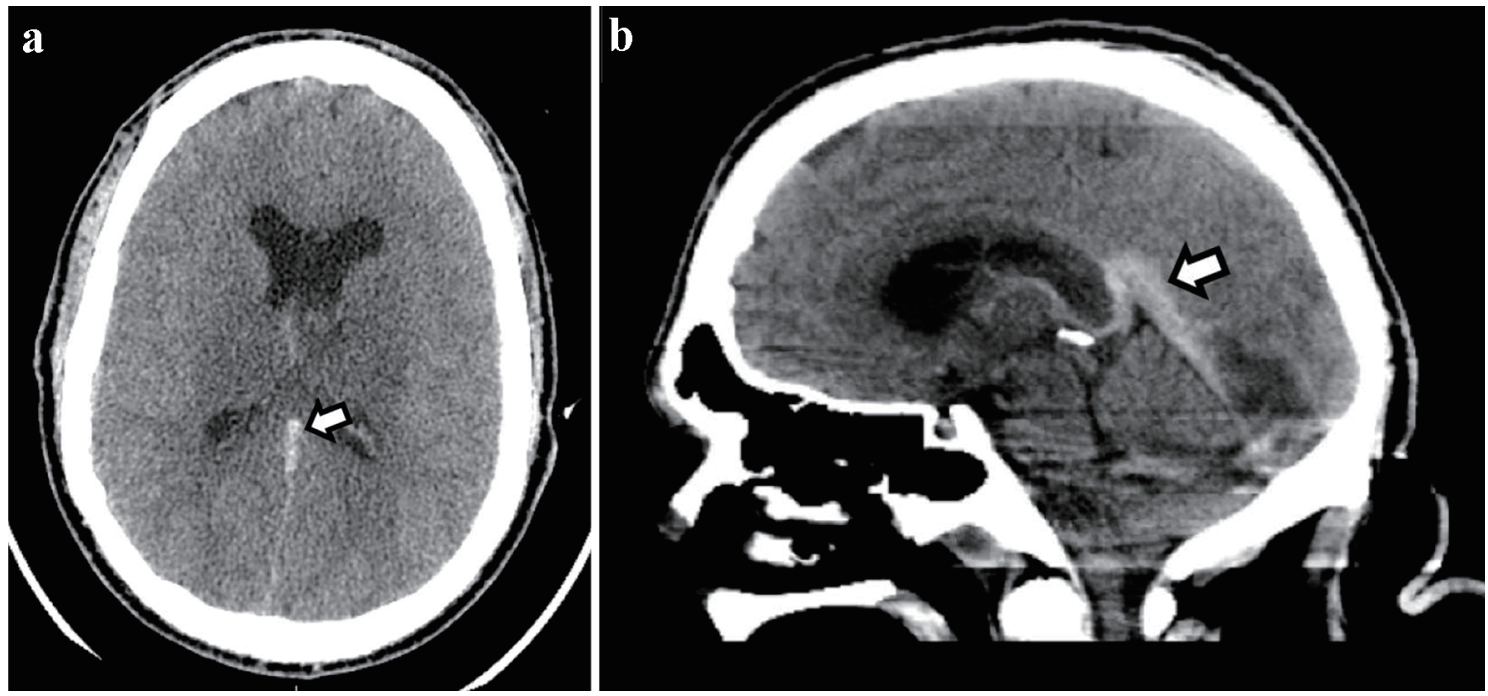

Figure 2. CT of the head without contrast ((a) axial and (b) sagittal)) shows a hyperdense straight sinus consistent with cerebral venous thrombosis. CT: computed tomography. 

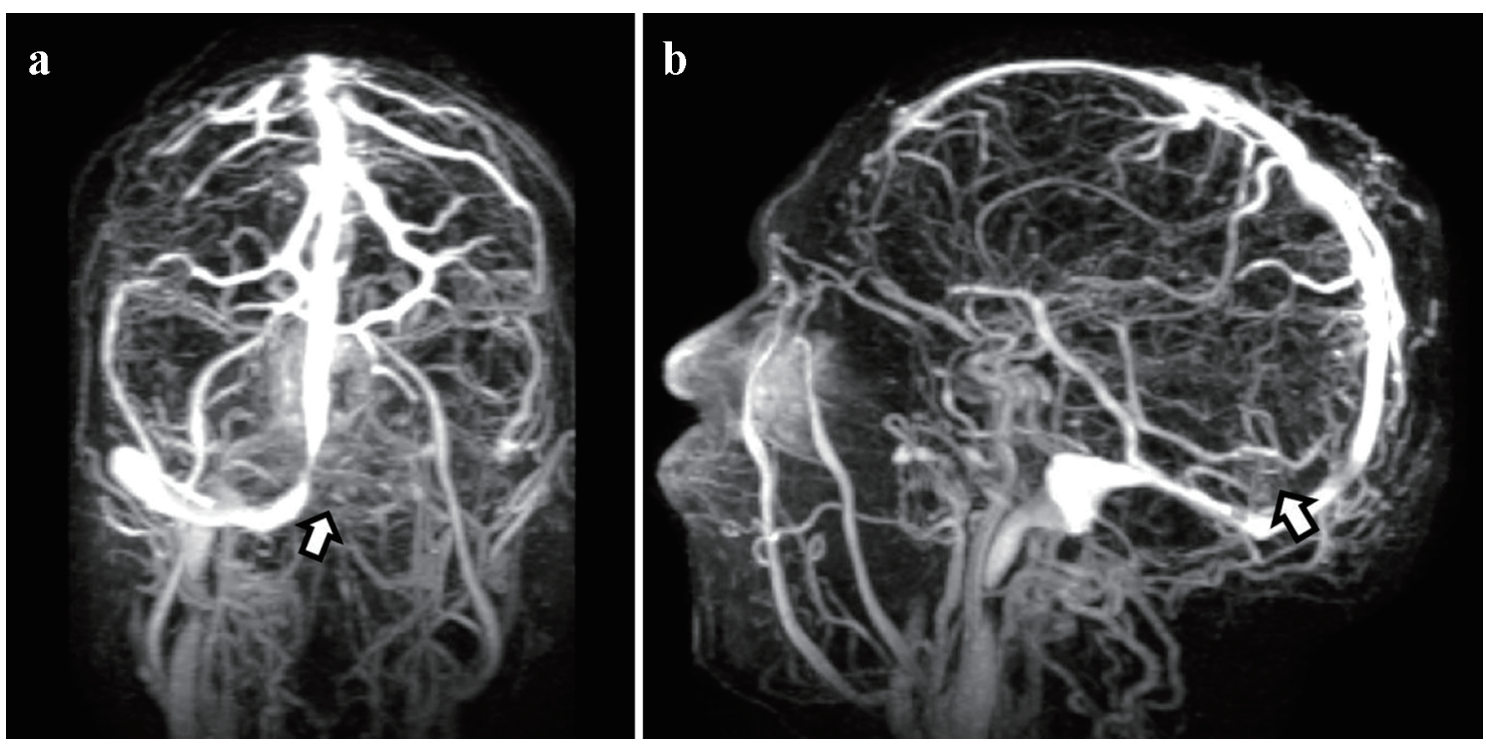

Figure 3. Magnetic resonance venography shows extensive dural venous sinus thrombosis. (a) Presence of occlusive thrombus on the left side involving the transverse, sigmoid, and superior aspect of the internal jugular vein. (b) Thrombosis of the vein of Galen and straight sinus.

discharged to a rehabilitation center on apixaban $5 \mathrm{mg}$ and levetiracetam $750 \mathrm{mg}$ every $12 \mathrm{~h}$. The patient's overall condition gradually improved with occupational, speech, and physical therapy prior to discharge. Given the unrevealing extensive workup, dural and cortical venous thrombosis was attributed to a hypercoagulable state induced by the underlying SARSCoV-2 infection.

\section{Case 2}

A right-handed 31-year-old man was found with severe de- creased level of consciousness after a fall and transported to the ED of a local hospital via the emergency medical squad. Past medical history was significant for major depressive disorder, autism, and a recent SARS-CoV-2 infection diagnosed 12 days prior. Home prescribed medications included bupropion. Social history was unrevealing for any use of alcohol or recreational drugs. Family history was noncontributory. Review of system provided by the family was positive for decreased oral intake for the past $24 \mathrm{~h}$, shortness of breath, and a recent fall. On initial evaluation, the patient was afebrile but appeared ill with clinical evidence of severe dehydration. Initial CT of the head revealed subarachnoid hemorrhage over
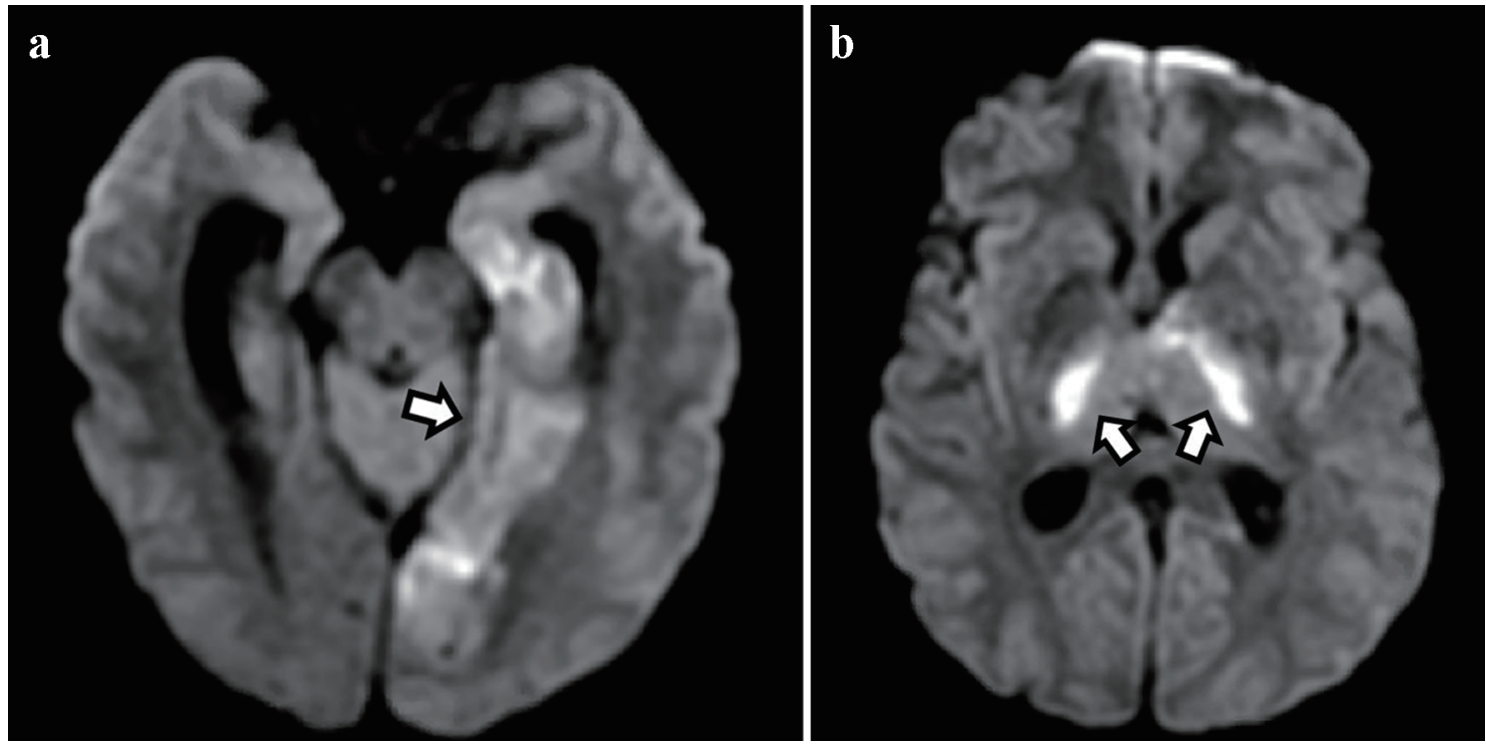

Figure 4. Diffusion-weighted imaging shows areas of restricted diffusion in the medial temporal and occipital lobes (a) and bilateral thalami (b) consistent with acute ischemic stroke. 


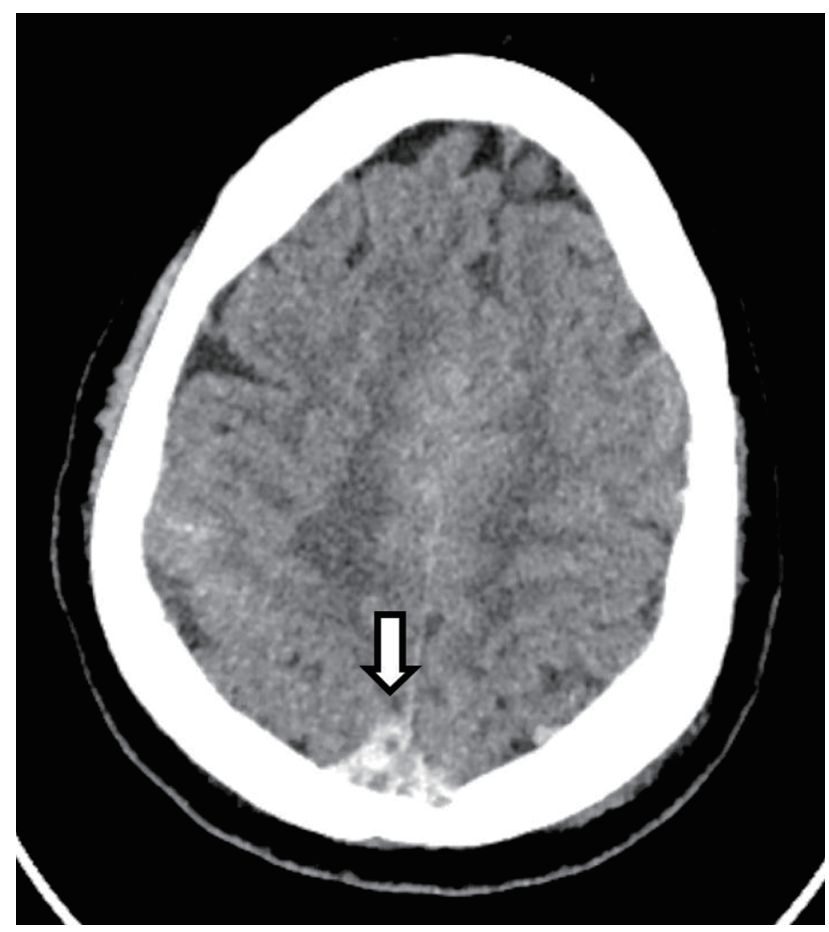

Figure 5. CT of the head without contrast shows an area of hyperdensity involving the superior sagittal sinus (arrow), consistent with a thrombus. CT: computed tomography.

the right convexity (images not shown). In the ED, the patient had continuous uncontrolled movements of the right upper and lower extremities followed by a grand-mal seizure of a 5-min duration. Patient was treated with $2 \mathrm{mg}$ of IV lorazepam and loaded with $2 \mathrm{~g}$ of levetiracetam. He was further treated with paralytic and sedative agents and intubated for airway protection and consequently transferred to our neurocritical care unit for further management.

On initial evaluation, the patient was found to be severely somnolent with a GCS of 7. He was afebrile. General examination was unremarkable. Initial neurological evaluation revealed that patient was not following commands. Evaluation of fund of knowledge, memory, and judgement was limited due to decreased level of consciousness and the administration of sedatives. Cranial nerve examination revealed equal pupils with brisk reaction to light. Corneal, oculocephalic, gag and cough reflexes were intact. Patient demonstrated withdrawal to pain in all four extremities, and limited motor examination appeared non-focal. Coordination and gait examination could not be assessed.

Laboratory workup included a CBC with a WBC of 13.7 $\times 10^{3} / \mathrm{mm}^{3}$ (normal range: $4.5-11 \times 10^{3} / \mathrm{mm}^{3}$ ). CMP revealed serum glucose level of $136 \mathrm{mg} / \mathrm{dL}$ (normal range: 70 - 110 $\mathrm{mg} / \mathrm{dL}$ ), albumin of $3.4 \mathrm{~g} / \mathrm{dL}$ (normal range: $3.50-5.0 \mathrm{~g} / \mathrm{dL}$ ), total bilirubin of $1.2 \mathrm{mg} / \mathrm{dL}$ (normal range: $0.2-1.0 \mathrm{mg} / \mathrm{dL}$ ), and a serum anion gap of 16 (normal range: 3 - 11). Additional laboratory workup revealed elevated fibrinogen of $594 \mathrm{mg} / \mathrm{dL}$ (normal range: 180 - 500), D-dimer of $8.58 \mu \mathrm{g} / \mathrm{mL}$ (normal $<0.5 \mu \mathrm{g} / \mathrm{mL}$ ), and C-reactive protein of $87.4 \mathrm{mg} / \mathrm{L}$ (normal range: $0.0-10.0 \mathrm{mg} / \mathrm{L}$ ). Other normal investigations included
RBC, platelet count, hemoglobin, hematocrit, BUN, creatinine, sodium, potassium, chloride, calcium, partial thromboplastin time, and prothrombin time.

Chest X-ray showed hazy opacities of the lateral left midlung zone. CT of the chest with contrast revealed atypical pneumonitis consistent with the recent SARS-CoV-2 infection but no evidence of pulmonary embolism. Repeated CT of the head revealed areas of hyperattenuation in several portions of the superior sagittal sinus (Fig. 5) as well as expansion of several cortical veins, raising the suspicion for dural venous sinus and cortical venous thromboses (images not shown). CT of the head also showed slight enlargement of the previously noted subarachnoid hemorrhage (SAH) and right-sided sulci effacement of the parietal and temporal lobes (images not shown). Further imaging studies with brain MRI and MRV of the head revealed extensive thromboses in the distal superior sagittal sinus, right transverse and sigmoid sinuses, and multiple cortical veins (Fig. 6a, b). The left transverse and sagittal sinuses and bilateral internal jugular veins were patent. The patient was started on continuous IV heparin infusion. Complete inherited and acquired thrombotic risk panel including factor $\mathrm{V}$ Leiden and prothrombin gene mutations was unremarkable. The patient's overall condition gradually improved, and he was eventually transferred out of the neurocritical unit. IV heparin was transitioned to apixaban, $5 \mathrm{mg}$ every $12 \mathrm{~h}$. Antiepileptic treatment was resumed with levetiracetam $500 \mathrm{mg}$ every $12 \mathrm{~h}$ with no recurrence of seizures. Given the unrevealing extensive workup, dural and cortical venous thrombosis was attributed to a hypercoagulable state likely elicited by the current SARSCoV-2 infection.

\section{Discussion}

\section{Epidemiology}

The World Health Organization recognized the SARS-CoV-2 pandemic after a cluster of cases with atypical respiratory symptoms were reported in Wuhan, China [2]. Since then, our knowledge regarding the multisystem involvement of SARS-CoV-2 has grown significantly. An earlier study reported that nearly $36 \%$ of patients with SARS-CoV-2 infection have underlying neurological manifestations [3]. Neurological manifestations of SARS-CoV-2 were classified into three categories, based on involvement of the central nervous system, peripheral nervous system, or skeletal muscle [3]. Of the multiple central nervous system manifestations of SARS-CoV-2, acute cerebrovascular events including CVST have been reported.

CVST is a rare cause of stroke in young adults, with an estimated annual incidence of 1.3 - 1.6/100,000 during the preCOVID era [4], and it constitutes up to $12 \%$ of unusual causes of ischemic stroke according to one case series [5]. Numerous conditions have been demonstrated to increase the risk of CVST [4]. It is worth noting that hematologic disorders are the most common but under-recognized causes of CVST [4, 6]. During the ongoing SARS-CoV-2 pandemic, there is a significant increase in the incidence of CVST with concomitant 

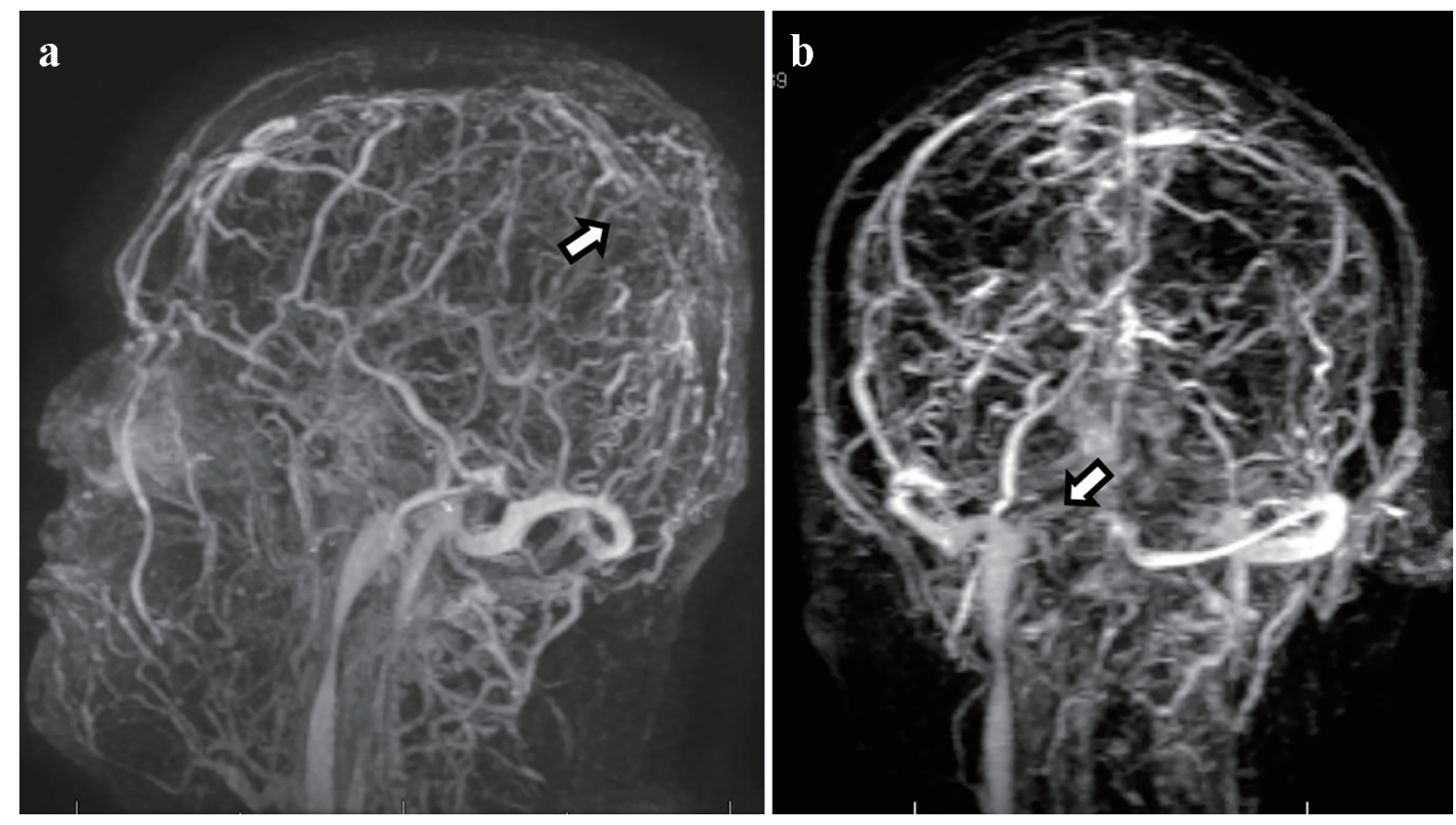

Figure 6. Magnetic resonance venography shows extensive dural venous thrombosis involving multiple transcortical veins along the high convexities, distal two-thirds of the superior sagittal sinus (a), and right-sided transverse and sigmoid sinuses (b).

infection of approximately $4.5-20 / 100,000$ [7, 8], up to 15 times greater than that without the infection.

Patients with CVST and underlying SARS-CoV-2 infection reported in the literature were often younger with a mean age of $43.6 \pm 17.6$ years [7]. An alternative multicenter study reported a mean age of patients with CVST and SARS-CoV-2 of 63 years and suggested that age plays a minor role as a risk factor, as up to $75 \%$ had no known risk factors for thrombotic disease [9]. Both our patients are young men with no known risk factors for venous thromboembolism and unrevealing workup for inherited and acquired thrombotic disorders. Historically, CVST is more common in women than men, likely due to gender-specific risk factors such as oral contraceptives use, hormone replacement therapy, pregnancy, and puerperium [10]. Conversely, recent studies report that gender does not play a significant role in SARS-CoV-2-associated CVST with men being affected equally or more in comparison to women $[7,9]$. A similar observation is made in our report with both patients being men with age between 30 and 40 years.

\section{Clinical presentation}

Patients with CVST and underlying SARS-CoV-2 infection have been reported to present with mild or no respiratory symptoms [7]. The diagnosis of COVID-19 in case 2 was made nearly 2 weeks prior to the diagnosis of CVST, with no respiratory symptoms on initial presentation. Our series suggest that CVST may occur regardless of the degree of severity of the infection and in the absence of respiratory symptoms, like observations made previously $[7,9,11]$.

In general, headache is the most common symptom in approximately $85-95 \%$ of patients with CVST followed by fo- cal neurological symptoms $[12,13]$. In one study, headache in CVST patients with an underlying SARS-CoV-2 infection was reported in approximately $83 \%$ of patients with decreased level of consciousness in $40 \%$ of all cases [11]. Other reported symptoms were seizures and focal neurological deficits [7, 11]. Both our patients initially presented with altered level of consciousness with no reported headache but developed seizures during their hospitalization.

The superior sagittal sinus is the most involved in patient with CVST. CVST in patients with concomitant SARS-CoV-2 seems to commonly involve other sinuses as well as the deep venous system including the vein of Galen, internal and deep medullar veins $[7,9,14]$. Imaging modalities including noncontrast CT, MRI, and MRV are recommended in patients with a clinical presentation suggestive of CVST. CVST was often associated with hemorrhagic venous infarctions with equivalent involvement of deep and superficial veins [15]. Isolated or multiple venous involvement has been reported in addition to arterial stroke and intraparenchymal hemorrhage.

\section{Pathophysiology}

SARS-CoV-2 has been associated with both arterial and venous thrombotic events, with intracranial hemorrhage rarely reported. The incidence of thrombotic events in intensive care unit (ICU) patients with SARS-CoV-2 was reported to be approximately $22-50 \%$, with predominance of venous over arterial thromboembolism $[16,17]$. The virus has the potential to gain entry into cells by interacting with the angiotensinconverting enzyme-2 (ACE-2) receptor [18]. This interaction can ultimately lead to endothelial dysfunction [19], triggering a cascade of thrombosis and ultimately vascular throm- 
botic events. The multiorgan expression of ACE-2 receptors in kidneys, liver, heart, and brain explains the extrapulmonary thrombotic complications of SARS-CoV-2 in addition to triggering a pro-inflammatory milieu that shifts homeostasis $[20$, 21].

The hypercoagulable state associated with SARS-CoV-2 is mediated by elevated acute phase reactants and circulating prothrombotic compounds such as fibrinogen, D-dimer, and antiphospholipid antibodies [22-26]. Elevated levels of Ddimer and fibrinogen have been reported in critically ill patients with SARS-CoV-2, contributing to the underlying hypercoagulability state associated with the infection [27]. The inflammatory response also triggers the release of other clotting factors including factor VIII and von Willebrand factor [28]. Overall, the inflammatory disease process and associated cytokine storm, which is most prominent in the late stages of the infection, play a major role in mediating various thrombotic events [23, 24]. An even higher risk of thrombotic events associated with SARS-CoV-2 can be associated with advanced age, obesity, immobility, recent trauma or surgery, and/or underlying active malignancy [20].

\section{Treatment}

CVST manifests as symptoms and signs of intracranial hypertension, encephalopathy and/or focal neurological deficits [20]. Admission to the neurocritical care unit with close monitoring and management of elevated intracranial pressure is recommended. Anticoagulation coupled with hydration seems to be the most effective pharmacotherapy in patients with CVST associated with an underlying SARS-CoV-2 infection, even in the presence of hemorrhage. In addition to its anti-inflammatory and anticoagulation effects, heparin has the potential to interfere with viral entry into cells by binding to certain surface proteins [28]. The duration of anticoagulation however is uncertain as no specific guidelines exist. In the absence of seizures, prophylactic anti-epileptic therapy for CVST is not recommended [20]. The role of endovascular intervention in CVST patients with SARS-CoV-2 is unknown given the limited number of cases reported in the literature [29, 30].

\section{Prognosis}

The prognosis of patients with concomitant CVST and SARSCoV-2 infection appears to be worse than without the underlying infection [7]. The mortality rate of SARS-CoV-2 infection during the pandemic was estimated to be $5.7 \%$ as of March 2020 [31]. Studies report a remarkably high mortality rate ranging from $12.5 \%$ to $45.5 \%$ in patients with CVST and recent SARS-CoV-2 infection [7,9], in contrast to $2-15 \%$ without the concomitant infection $[4,14,32]$. In one systemic review, an unusually high rate of mortality of $45 \%$ was reported [5]. This may be related to the extensiveness and anatomical location of the venous thrombosis, with deep venous system involvement associated with a higher mortality rate [7, 14]. Both our patients were discharged without any residual neu- rological deficits, suggesting that young age and absence of other underlying comorbidities may contribute to a more favorable prognosis. Bolagi et al observed that early treatment with anti-coagulants and anti-epileptic medications is associated with a good functional outcome despite the severity of neurological deficits [33]. D-dimer level is one of the most important prognostication factors for patients with COVID-19 infection and thrombotic events, as higher levels are thought to correlate with clot burden [23].

\section{Conclusion}

Our case series serve as additional evidence to the growing body of literature that suggests SARS-CoV-2 infection and CVST likely have a causal relationship. A significant increase in the incidence of CVST among patients with a recent SARSCoV-2 infection has been noted, particularly in the young population, constituting a potentially devastating neurological sequela in this patient population. The prognosis in patients with CVST and SARS-CoV-2 infection appears less favorable than without the coexisting infection, and early clinical recognition, diagnosis, and anticoagulation is strongly recommended.

\section{Acknowledgments}

Our warmest thanks go to the patient for allowing us to report this case.

\section{Financial Disclosure}

This research received no specific grant from any funding agency in the public, commercial, or not-for-profit sectors.

\section{Conflict of Interest}

The authors report no conflict of interest.

\section{Informed Consent}

Informed consent to write and publish the case was obtained from the patients.

\section{Author Contributions}

MF provided the body of the case report, and summarized the case. ML provided the body of the case report, and summarized the case. AR drafted the discussion, and revised the final draft. RCA edited the figures and participated in revising the final draft. ER drafted the discussion, participated in revising the final draft and submitted the final paper for review. HY revised the final draft and provided expertise on the clinical significance of the case. 


\section{Data Availability}

The authors declare that the data supporting the findings of this study are available within the article.

\section{References}

1. Devasagayam S, Wyatt B, Leyden J, Kleinig T. Cerebral venous sinus thrombosis incidence is higher than previously thought: a retrospective population-based study. Stroke. 2016;47(9):2180-2182.

2. WHO. Timeline of WHO's response to COVID-19. World Health Organization. Published 2020. https://www.who. int/emergencies/diseases/novel-coronavirus-2019/interactive-timeline.

3. Mao L, Jin H, Wang M, Hu Y, Chen S, He Q, Chang J, et al. Neurologic manifestations of hospitalized patients with coronavirus disease 2019 in Wuhan, China. JAMA Neurol. 2020;77(6):683-690.

4. Silvis SM, de Sousa DA, Ferro JM, Coutinho JM. Cerebral venous thrombosis. Nat Rev Neurol. 2017;13(9):555565 .

5. Arboix A, Bechich S, Oliveres M, Garcia-Eroles L, Massons J, Targa C. Ischemic stroke of unusual cause: clinical features, etiology and outcome. Eur J Neurol. 2001;8(2):133-139.

6. Arboix A, Jimenez C, Massons J, Parra O, Besses C. Hematological disorders: a commonly unrecognized cause of acute stroke. Expert Rev Hematol. 2016;9(9):891-901.

7. Tu TM, Goh C, Tan YK, Leow AS, Pang YZ, Chien J, Shafi $\mathrm{H}$, et al. Cerebral venous thrombosis in patients with COVID-19 infection: a case series and systematic review. J Stroke Cerebrovasc Dis. 2020;29(12):105379.

8. Siegler JE, Cardona P, Arenillas JF, Talavera B, Guillen AN, Chavarria-Miranda A, de Lera M, et al. Cerebrovascular events and outcomes in hospitalized patients with COVID-19: The SVIN COVID-19 Multinational Registry. Int J Stroke. 2021;16(4):437-447.

9. Abdalkader M, Shaikh SP, Siegler JE, Cervantes-Arslanian AM, Tiu C, Radu RA, Tiu VE, et al. Cerebral venous sinus thrombosis in COVID-19 patients: a multicenter study and review of literature. J Stroke Cerebrovasc Dis. 2021;30(6):105733.

10. Coutinho JM, Ferro JM, Canhao P, Barinagarrementeria F, Cantu C, Bousser MG, Stam J. Cerebral venous and sinus thrombosis in women. Stroke. 2009;40(7):2356-2361.

11. Mowla A, Shakibajahromi B, Shahjouei S, BorhaniHaghighi A, Rahimian N, Baharvahdat H, Naderi S, et al. Cerebral venous sinus thrombosis associated with SARS-CoV-2; a multinational case series. J Neurol Sci. 2020;419:117183.

12. Wang JW, Li JP, Song YL, Tan K, Wang Y, Li T, Guo $\mathrm{P}$, et al. Clinical characteristics of cerebral venous sinus thrombosis. Neurosciences (Riyadh). 2015;20(3):292295.

13. Shakibajahromi B, Haghighi AB, Salehi A, Vardanjani HM, Ghaedian M, Safari A, Mowla A. Clinical and ra- diological characteristics and predictors of outcome of cerebral venous sinus thrombosis, a hospital-based study. Acta Neurol Belg. 2020;120(4):845-852.

14. Ferro JM, Canhao P, Stam J, Bousser MG, Barinagarrementeria F, Investigators I. Prognosis of cerebral vein and dural sinus thrombosis: results of the International Study on Cerebral Vein and Dural Sinus Thrombosis (ISCVT). Stroke. 2004;35(3):664-670.

15. Ladopoulos T, Zand R, Shahjouei S, Chang JJ, Motte J, Charles James J, Katsanos AH, et al. COVID-19: Neuroimaging Features of a Pandemic. J Neuroimaging. 2021;31(2):228-243.

16. Artifoni M, Danic G, Gautier G, Gicquel P, Boutoille D, Raffi F, Neel A, et al. Systematic assessment of venous thromboembolism in COVID-19 patients receiving thromboprophylaxis: incidence and role of Ddimer as predictive factors. J Thromb Thrombolysis. 2020;50(1):211-216.

17. Edler C, Schroder AS, Aepfelbacher M, Fitzek A, Heinemann A, Heinrich F, Klein A, et al. Dying with SARSCoV-2 infection-an autopsy study of the first consecutive 80 cases in Hamburg, Germany. Int J Legal Med. 2020;134(4):1275-1284.

18. Khan IH, Zahra SA, Zaim S, Harky A. At the heart of COVID-19. J Card Surg. 2020;35(6):1287-1294.

19. Escher R, Breakey N, Lammle B. Severe COVID-19 infection associated with endothelial activation. Thromb Res. 2020;190:62.

20. Loo J, Spittle DA, Newnham M. COVID-19, immunothrombosis and venous thromboembolism: biological mechanisms. Thorax. 2021;76(4):412-420.

21. Puelles VG, Lutgehetmann M, Lindenmeyer MT, Sperhake JP, Wong MN, Allweiss L, Chilla S, et al. Multiorgan and renal tropism of SARS-CoV-2. N Engl J Med. 2020;383(6):590-592.

22. Khan IH, Savarimuthu S, Leung MST, Harky A. The need to manage the risk of thromboembolism in COVID-19 patients. J Vasc Surg. 2020;72(3):799-804.

23. Griffin DO, Jensen A, Khan M, Chin J, Chin K, Saad J, Parnell R, et al. Pulmonary Embolism and Increased Levels of d-Dimer in Patients with Coronavirus Disease. Emerg Infect Dis. 2020;26(8):1941-1943.

24. Panigada M, Bottino N, Tagliabue P, Grasselli G, Novembrino C, Chantarangkul V, Pesenti A, et al. Hypercoagulability of COVID-19 patients in intensive care unit: A report of thromboelastography findings and other parameters of hemostasis. J Thromb Haemost. 2020;18(7):1738-1742.

25. Valderrama EV, Humbert K, Lord A, Frontera J, Yaghi S. Severe Acute Respiratory Syndrome Coronavirus 2 Infection and Ischemic Stroke. Stroke. 2020;51(7):e124e127.

26. Zhang Y, Xiao M, Zhang S, Xia P, Cao W, Jiang W, Chen H, et al. Coagulopathy and Antiphospholipid Antibodies in Patients with Covid-19. N Engl J Med. 2020;382(17):e38.

27. Han H, Yang L, Liu R, Liu F, Wu KL, Li J, Liu XH, et al. Prominent changes in blood coagulation of patients with SARS-CoV-2 infection. Clin Chem Lab Med. 2020;58(7):1116-1120. 
28. Sholzberg M. COVID-19 and hypercoagulability. Clin Adv Hematol Oncol. 2020;18(7):386-389.

29. Ostovan VR, Foroughi R, Rostami M, Almasi-Dooghaee M, Esmaili M, Bidaki AA, Behzadi Z, et al. Cerebral venous sinus thrombosis associated with COVID-19: a case series and literature review. J Neurol. 2021.

30. Abouhashem S, Eldawoody H, Taha MM. Cerebral venous sinus thrombosis in patients with COVID-19 infection. Interdiscip Neurosurg. 2021;24:101091.
31. Baud D, Qi X, Nielsen-Saines K, Musso D, Pomar L, Favre G. Real estimates of mortality following COVID-19 infection. Lancet Infect Dis. 2020;20(7):773.

32. Bousser MG, Ferro JM. Cerebral venous thrombosis: an update. Lancet Neurol. 2007;6(2):162-170.

33. Bolaji P, Kukoyi B, Ahmad N, Wharton C. Extensive cerebral venous sinus thrombosis: a potential complication in a patient with COVID-19 disease. BMJ Case Rep. 2020;13(8):e236820. 